\title{
Identfication of Phase I and Phase II Metabolites of Hesperetin in Rat Liver Microsomes by Liquid Chromatography-Electrospray Ionization-Tandem Mass Spectrometry
}

\author{
Unyong Kim ${ }^{\text {a,b }}$, Sang Beom Han ${ }^{\mathrm{a}}$, Oh-Seung Kwon ${ }^{\mathrm{b}}$, and Hye Hyun Yoo ${ }^{\mathrm{c}, *}$ \\ ${ }^{a}$ Department of Pharmaceutical analysis, College of Pharmacy, Chung-Ang university, Seoul, Korea \\ ${ }^{b}$ Doping Control Center, Korea institute of Science and Technology \\ ${ }^{c}$ Department of Pharmacy, College of Pharmacy, Hanyang University, Ansan, Gyeonggi-do, Korea
}

Received February 23, 2011; Revised March 12, 2011; Accepted March 12, 2011

First published on the web March 15, 2011; DOI: 10.5478/MSL.2011.2.1.020

\begin{abstract}
The purpose of this study is to investigate the in vitro metabolism of hesperetin, a bioflavonoid. Hesperetin was incubated with rat liver microsomes in the presence of NADPH and UDP-glucuronic acid for $30 \mathrm{~min}$. The reaction mixture was analyzed by liquid chromatography-ion trap mass spectrometer and the chemical structures of hesperetin metabolites were characterzed based on their MS/MS spectra. As a result, a total of five metabolites were detected in rat liver microsomes. The metabolites were identified as a de-methylated metabolite (eriodictyol), two hesperetin glucuronides, and two eriodictyol glucuronides.
\end{abstract}

Key words: Hesperetin, in vitro Metabolism, Rat Liver Microsomes, LC/MS

\section{Introduction}

Hesperidin, a flavanone glycoside, is the major chemical constituent in the citrus fruits. ${ }^{1-3}$ Hesperidin shows an antiinflammatroy effect by inhibition of eicosanoids synthesis. ${ }^{4,5}$ Other biological activities of hesperidin include blood cholesterol lowering effect, hypotensive effect, and protective effect against the sepsis. ${ }^{1-3,6,7}$ However, hesperidin itself does not penetrate the mucous membrane of the gastrointestinal (GI) tract, but hesperetin, an aglycone of hesperidin, is absorbed from the GI tract. ${ }^{1,8}$ Thus, hesperidin is converted to hesperetin by intestinal microflora and subsequently absorbed from intestinal mucosa ${ }^{1,3}$ to exert various biological activities in the body. In this context, the understanding of hesperetin metabolism is important to figure out pharmacokinetic behaviors and furthermore pharmacological effects of hesperidin. In this study, the metabolism of hesperetin in rat liver microsomes was characterized by LC-MS/MS analysis.

\section{Experimental}

\section{Reagents}

Hesperetin, glucose 6-phosphate, $\beta$-NADP+, glucose-6phosphate dehydrogenase, formic acid, dimethyl sulfoxide (DMSO), and UDP-glucuronic acid were purchased from Sigma Chemicals (St. Louis, MO). Rat liver microsomes

*Reprint requests to Dr. Hye Hyun Yoo

E-mail: yoohh@hanyang.ac.kr were purchased from BD gentest (Franklin Lakes, NJ, USA). High-performance liquid chromatography (HPLC)-grade acetonitrile and methanol were purchased from J. T. Baker (Phillipsburg, NJ). HPLC-grade water was prepared using a Milli-Q purification system (Millipore, Bedford, MA). The Oasis HLB cartridge (96-well plate) was purchased from Waters (Milford, MA). All other chemicals used were analytical grade.

\section{LC/MS/MS system}

The HPLC system consisted of an HP 1100 series binary pump HPLC system (Agilent, Palo Alto, CA) with an LC/MSD ion-trap mass spectrometer equipped with an electrospray ionization source. The column used for the separation was a Hydrosphere $\mathrm{C}_{18}(2 \times 150 \mathrm{~mm}, 5 \mu \mathrm{m}, \mathrm{YMC}$, Kyoto, Japan $)$. Column temperature was maintained at $35^{\circ} \mathrm{C}$ using a thermostatically controlled column oven. The HPLC mobile phases consisted of $0.1 \%$ formic acid (solvent A) and $90 \%$ ACN with $0.1 \%$ formic acid (solvent B). A gradient program was used for the HPLC separation at a flow rate of $0.2 \mathrm{~mL} / \mathrm{min}$. The mobile phase was initially composed of $10 \%$ solvent B for $3 \mathrm{~min}$, linearly programmed to $35 \%$ solvent $\mathrm{B}$ for $12 \mathrm{~min}$ and $90 \%$ solvent B for $15 \mathrm{~min}$, maintained for $5 \mathrm{~min}$, and changed back to the initial condition over $1 \mathrm{~min}$ followed by 9 min re-equilibration. The total run time was $45 \mathrm{~min}$. The entire column elutes were directly introduced into an electrospray ionization (ESI) interface. Nitrogen was used both as the nebulizing gas at $40 \mathrm{psi}$ and as the drying gas at a flow rate of $10 \mathrm{~L} / \mathrm{min}$. The drying temperature was $350^{\circ} \mathrm{C}$. The mass spectrometer was operated in positive ion mode in $\mathrm{m} / \mathrm{z}$ range 100-700. Helium was used as the collision gas for the 
tandem mass spectrometric experiments. Fragmentation was induced with resonant excitation amplitude of $0.9 \mathrm{~V}$.

\section{In vitro microsomal incubation}

Incubation mixtures consisted of $0.5 \mathrm{mg} / \mathrm{mL}$ rat liver microsomes, $50 \mu \mathrm{M}$ hesperetin, and an NADPH-generating system (NGS; $0.1 \mathrm{M}$ glucose-6-phosphate, $10 \mathrm{mg} / \mathrm{mL} \mathrm{NADP+}$, and $1 \mathrm{U} / \mathrm{mL}$ glucose-6-phosphate dehydrogenase) in a total volume of $200 \mu \mathrm{L}$ potassium phosphate buffer $(0.1 \mathrm{M}, \mathrm{pH} 7.4)$. To investigate the generation of glucuronide conjugates, $2 \mathrm{mg} / \mathrm{mL}$ UDP-glucuronic acid was added to the reaction mixture. Reactions were initiated by the addition of NGS after a $5 \mathrm{~min}$ pre-incubation at $37^{\circ} \mathrm{C}$. Incubations were carried out for $30 \mathrm{~min}$ and quenched by addition of $800 \mu \mathrm{L}$ ice cold $0.1 \%$ acetic acid to the reaction mixture. The samples were vortex-mixed for $1 \mathrm{~min}$ and applied to the OASIS HLB cartridges (Waters, Milford, MA) which were activated with $1 \mathrm{~mL}$ of $\mathrm{MeOH}$ and equilibrated with $0.1 \%$ acetic acid. After sample loading, the cartridges were washed with $2 \mathrm{~mL}$ of $0.1 \%$ acetic acid $(1 \mathrm{~mL} \times 2)$ and eluted with $1 \mathrm{~mL}$ of $\mathrm{MeOH}$. The elutes were dried under $\mathrm{N}_{2}$ gas at $55^{\circ} \mathrm{C}$ and reconstituted with $100 \mu \mathrm{L}$ of $70 \% \mathrm{MeOH}$. Five microliter-aliquots were injected onto the LC-MS/MS system.

\section{Results and Discussion}

The extracted ion chromatograms (EICs) of hesperetin and its metabolites in rat liver microsomes were shown in Figure 1. When hesperetin was incubated with rat liver microsomes in the presence of NADPH, hesperetin was metabolized to yield a demethylated metabolite, M1 (Figure 1A). Addition of UDP-glucuronic acid to the incubation mixure led to the generation of 4 more metabolites (M2 M5) from hesperetin which were considered as glucuronide conjugates (Figure 1B). The decrease in the peak intensities of hesperetin and M1 in Figure 2B indicates that M2, M3, M4, and M5 were generated from hesperetin and M1. In Figure 1A, two peaks aside from M1 shown in the EIC of m/z 289 (at 24 25 min), and one peak in the EIC of m/z 465 (at $21 \mathrm{~min}$ ) were thought to be origainated from the matrix, which were also detected in the EIC of the UDP-glucuronic acid-added mixture (Figure 1B).

To characterize the chemical structure of hesperetin metabolites, MS/MS experiments were performed. Based on the MS/MS fragmnetation patterns, the chemical structures of the metabolites were elucidated (Figure 2). Hesperetin was observed at the retention time of 26.2 with a protonated ion at $\mathrm{m} / \mathrm{z} 303$. In the collision-induced dissociation (CID) spectra of hesperetin, the fragment ion of $\mathrm{m} / \mathrm{z} 177$ was produced predominantly. The ion of $\mathrm{m} / \mathrm{z} 177$ was formed by cross ring cleavage of 1,4-dioxane ring. The ion of $\mathrm{m} / \mathrm{z} 153$ was formed by the retro diel-alder (RDA) type cleavage. The catechol moiety was released from $\mathrm{C}$ ring in the CID process and formed fragment ion of $\mathrm{m} / \mathrm{z} 179$. Generally, cross ring cleavage of 1,4-dioxane ring, a)

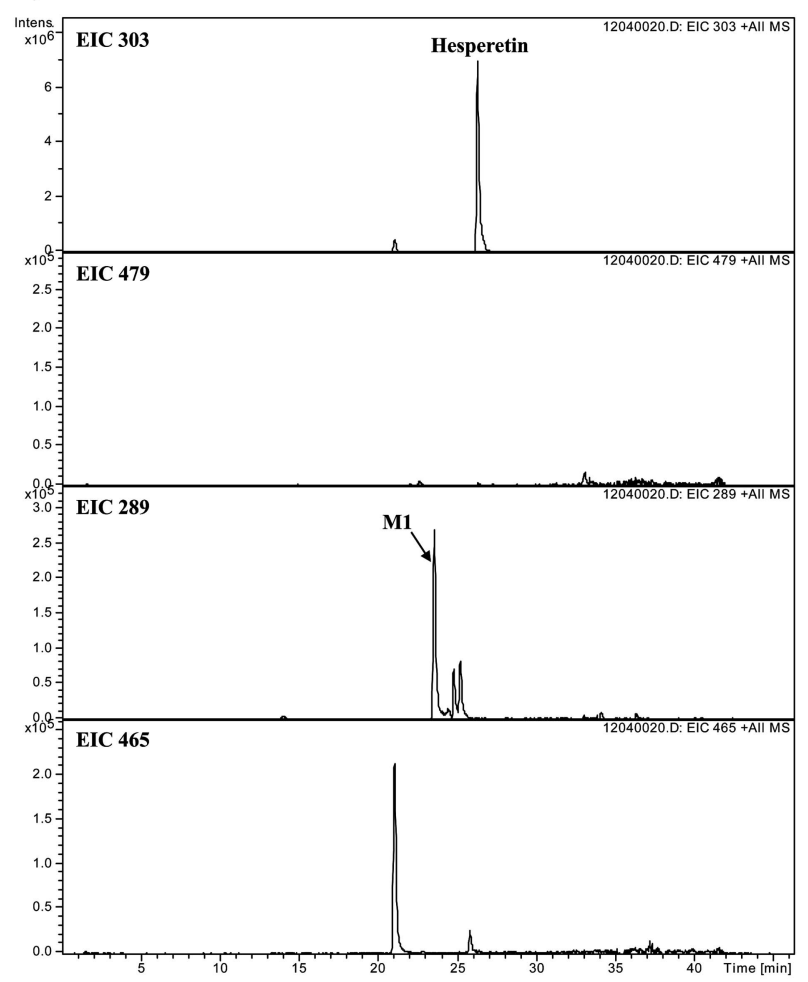

b)

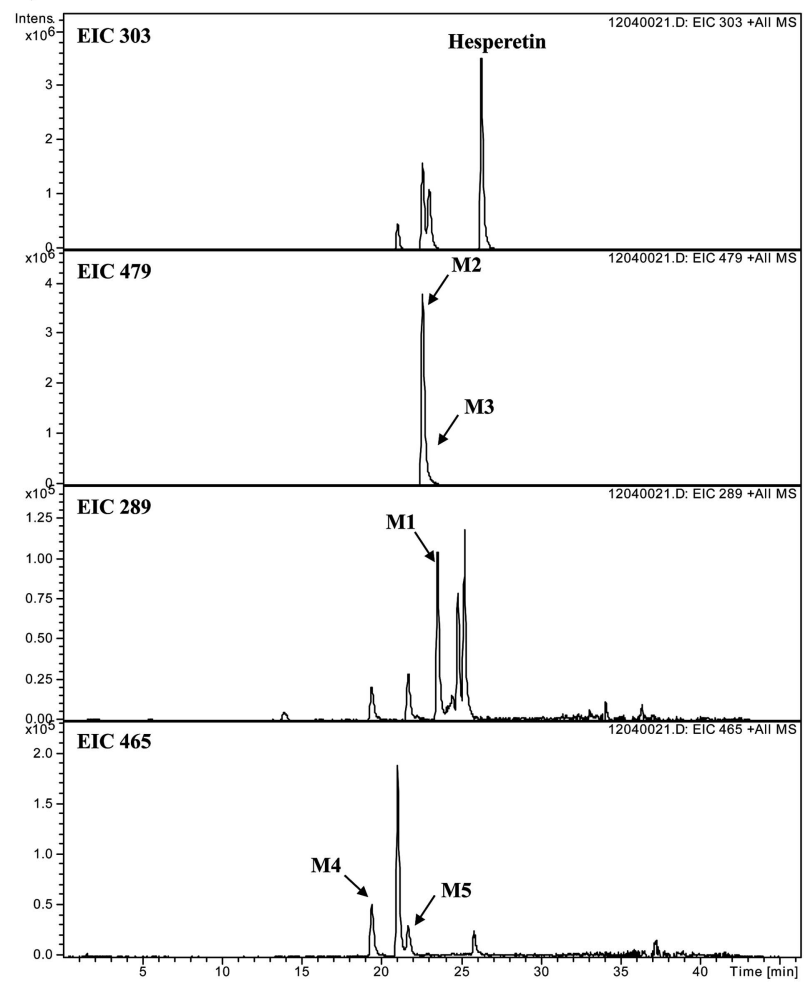

Figure 1. Extracted ion chromatograms (EICs) for hesperetin and its metabolites. Incubation mixtures without (A) and with (B) UDPglucuronic acid. 

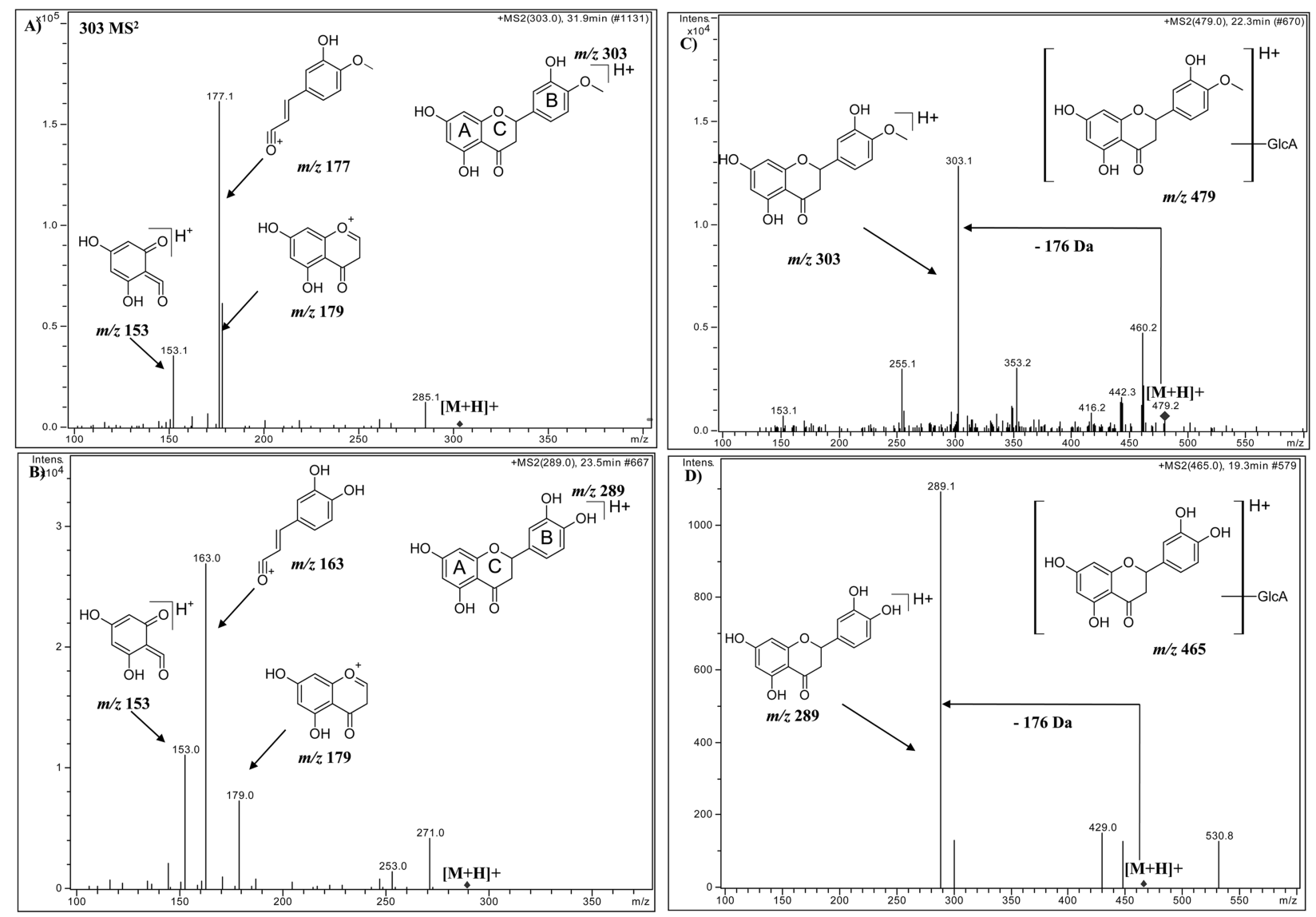

Figure 2. Collision-induced dissociation (CID) mass spectra of hesperetin (A), eriodictyol (M1)(B), hesperetin glucuronides (M2 and $\mathrm{M} 3)(\mathrm{C})$, and eriodictyol glucuronides (M4 and M5)(D).

RDA type cleavage, and release of B-ring are known to be the common fragmentation processes found in the CID process of flavonoids. $^{9-11}$ Such fragmnetation patterns were also observed in the $\mathrm{MS}^{2}$ spectra of hesperetin in this study.

The $[\mathrm{M}+\mathrm{H}]^{+}$ion of $\mathrm{M} 1$ was observed at $\mathrm{m} / \mathrm{z} 289$ and its major product ions were observed at $\mathrm{m} / \mathrm{z} 179, \mathrm{~m} / \mathrm{z} 163$, and $\mathrm{m} / \mathrm{z} 153$. The fragmentation pattern of M1 was similar to that of hesperetin. For example, the fragment ion at $\mathrm{m} / \mathrm{z} 163$ was corresponding to that of hesperetin at $\mathrm{m} / \mathrm{z} 177$ which was formed by cross ring cleavage of 1,4-dioxane ring. Based on 14 da difference between M1 and hesperetin in $\mathrm{MS}^{2}$ spectra, $\mathrm{M} 1$ was characterized as a $O$-demethylate metabolite, namely eriodictyol based on the literature. .,12 $^{32}$

The metabolites M2 and M3 were observed at 22.5 and $22.9 \mathrm{~min}$, respectively, with protonated ions at $\mathrm{m} / \mathrm{z} 479$. M2 and M3 were not separated clearly in the EIC of m/z 479 . However, in the EIC of $\mathrm{m} / \mathrm{z} 303$, two peaks were observed at the same retention time as M2 and M3, which may be the product ion peaks generated by the CID of ions at $\mathrm{m} / \mathrm{z} 479$. This revealed that M2 and M3 are the isobaric metabolites of hesperetin. The characteristic loss of 176 da in glucuroinide conjugates was found in the $\mathrm{MS}^{2}$ spectra of protonated M2 and M3. That indicated M2 and M3 are the isobaric glucuronide conjugates of hesperetin.

The metabolites M4 and M5 were observed at 19.3 and $21.6 \mathrm{~min}$, respectively, with protonated ions at $\mathrm{m} / \mathrm{z} 465$. The molucular weight of M4 and M5 were 176 da higher than that of M1. The characteristic neutral loss of 176 da of glucuronic acid was also found in the $\mathrm{MS}^{2}$ spectra of protonated M4 and M5. Taken together, M4 and M5 were chracterized as the isobaric glucuronide conjugates of M1.

The present study showed that glucuronidation ocurred at different hydroxyl groups in hesperetin and M1 although the site of glucuronidation was not able to be identified. According to the literatures, ${ }^{6-13}$ the antioxidant potentials of flavonoids may be affected by the glucuronidation site. If glucuronidation occures at one of the hydroxyl groups in the B-ring, the reduction potential increased whereas antioxidant activity decreased. ${ }^{6,13}$ Thus, the site of glucuronidation in flavonoids could have a significant meaning in terms of antioxidant or free radical scavanging activities. Ameer et al. reported that the orally absorbed hesperetin was immediately metabolized to form glucuronide conjugates in the intestinal epithelium and liver. ${ }^{1}$ In our results obtained from the in vitro experiment, hesperetin glucuronide was found to be a major metabolite, which was in accordance to the in vivo 
metabolism data reported previously. ${ }^{1,3,14,15}$ In this study, we could identify the phase I and phase II metabolites of hesperetin using a simple in vitro metabolism system with rat liver microsomes and LC-MS/MS analysis.

\section{Acknowledgments}

This research was supported by a grant (09172KFDA996) from Korea Food \& Drug Administration in 2010.

\section{References}

1. Ameer, B.; Weintraub, R. A.; Johnson, J. V.; Yost, R. A.; Rouseff, R. L. Clin. Pharmacol. Ther. 1996, 60, 34.

2. Li, X. J. Pharm. Biomed. Anal. 2004, 34, 159.

3. Jin, M. J.; Kim, U.; Kim, I. S.; Kim, Y.; Kim, D.-H.; Han, S. B.; Kwon, O.-S.; Yoo, H. H. J. Toxicol. Environ. Health Part A 2010, 73, 1441.

4. Lonchampt, M.; Guardiola, B.; Sicot, N.; Bertrand, M.; Perdrix, L.; Duhault, J. Arzneimittelforschung 1989, 39, 882.

5. Jean, T.; Bodinier, M. C. Angiology 1994, 45, 554.

6. Spencer, J. P.; Chowrimootoo, G.; Choudhury, R.; Debnam, E. S.; Srai, S. K.; Rice-Evans, C. FEBS Lett. 1999, 458, 224.
7. Garg, A.; Garg, S.; Zaneveld, L. J.; Singla, A. K. Phytother. Res. 2001, 15, 655.

8. Kim, M.; Kometani, T.; Okada, S.; Shimuzu, M. Biosci. Biotechnol. Biochem. 1999, 63, 2183.

9. Kéki, S.; Tóth, K.; Zsuga, M.; Ferenczi, R.; Antus, S. Rapid Commun. Mass spectrom. 2007, 21, 2255.

10. Es-Safi, N. E.; Kerhoas, L.; Ducrot, P. H. Rapid Commun. Mass spectrom. 2005, 19, 2734.

11. Qi, J.; Xu, D.; Zhou, Y. F.; Qin, M. J.; Yu, B. Y. Rapid Commun. Mass spectrom. 2010, 24, 2193.

12. Nielsen, S. E.; Breinholt, V.; Justesen, U.; Cornett, C.; Dragsted, L. O. Xenobiotica 1998, 28, 389.

13. Tripoli, E.; La Guardia, M.; Giammanco, S.; Di Majo, D.; Giammanco, M. Food Chem. 2007, 104, 466.

14. Brand, W.; Boersma, M. G.; Bik, H.; Hoek-van den Hil, E. F.; Vervoort, J.; Barron, D.; Meinl, W.; Glatt, H.; Williamson, G; van Bladeren, P. J.; Rietjens, I. M. Drug Metab. Dispos. 2010, 38, 617.

15. Brand, W.; Shao, J.; Hoek-van den Hil, E. F.; van Elk, K. N.; Spenkelink, B.; de Haan, L. H.; Rein, M. J.; Dionisi, F.; Williamson, G.; van Bladeren, P. J.; Rietjens, I. M. J. Agric. Food Chem. 2010, 58, 6119. 